\title{
Methods for methods' sake
}

Welcome to Nature Methods, the latest addition to the Nature family! Over the past decade, eight Nature sister journals have been launched to fulfill demands, in specific scientific communities, for a quality journal dedicated to their discipline and combining primary research, opinions and news. The success of these journals has demonstrated the value of this approach. The creation of Nature Methods is based on a similar balance of content but responds to a slightly different need, expressed across multiple disciplines: the dissemination of innovative research methods for their own sake.

Our goal is to create a highly visible forum for the presentation of novel methods, because we believe that major methodological developments often take their roots in the cross-fertilization of different disciplines. By contrast, the 'methods sections' of specialized journals, although extremely useful for the targeted peer groups, do not grant technical information the wide exposure conducive to interdisciplinary exchanges. This journal, rather, addresses the broad audience of researchers working in the life sciences.

By putting a strong emphasis on the practical relevance of the journal's content, our aim is to provide researchers with new tools that can be readily adopted and translated into benefits for their own research. If our rallying phrase specifies "Techniques for life scientists and chemists," it is because biological research increasingly benefits from the input of technologies that were born and enjoyed a full life in chemistry labs. The growing use of mass spectrometry to study the proteome, new chemical sensors to observe cellular processes, and novel crosslinkers to discover the unknown relationships between enzymes and substrates are only a few examples of the impact that chemistry has had on modern biology. It thus seems important to bridge the two communities, bringing chemistry tools within the sight and hands of biologists and making chemists aware of biologists' needs.

Hitherto, the methodological literature has been primarily dedicated to techniques that have already gained popularity. Nature Methods will make use of the periodical format to introduce methods at a stage where they have not yet gained a track record of success but show definite promise and are likely to stimulate other researchers. However, methodological developments can be very incremental and even the avid technical reader can easily become overwhelmed by information. To avoid this dilution effect, our aim is to publish a relatively small number of papers representing major methodological developments that are likely to be influential. In the tradition of Nature journals, this selection relies on a thorough peer review process.

But established methods will also be addressed. The unique Protocol section will focus on established methods that still present a technical challenge. Nature Methods is producing this section in association with Cold Spring Harbor Laboratory Press, the experienced and trusted publisher of numerous laboratory manuals that constitute the prime references in their fields. This synergy between two editorial teams will bring quality procedures to a multitude of benches every month. In a similar spirit, the Reviews section will attempt to address challenges that arise when the general direction of research in a field makes certain recently developed methods almost indispensable. To this end, we are asking specialists to raise practical issues and provide guidance to newcomers wishing to integrate a given technology into their research program. In addition, all the 'front-end' sections that you have come to expect in a Nature journal will cater to the needs of a general audience.

It is stating a stereotype to say that methodology is a driving force of scientific progress. Most will agree, too, that the birth of a new method comes only with the painstaking accumulation of optimization and validation. For a bold young researcher willing to venture into the creation of a significantly novel tool, the effort-consuming exercise of method development can easily take up most of a typical postdoctoral project. Sadly, unless the successful application of the new tool quickly results in a key biological insight that opens the door to a major publication, this work amounts to what might be considered 'just' a methods paper. The unfortunate reality is that technical creativity is not always rewarded for its own, significant value and that the current publication landscape, by providing little incentive for description of novel techniques, makes the qualifier 'methods' an almost dismissive term.

We want to change this attitude. We hope that you will work with us to make this journal into a prime tool for the research community, whose quality will make it rewarding to publish methods papers . 